\title{
Analysis of Spatial Sensitivity Based on Electrostatic Monitoring Technique in Oil-Lubricated System
}

\author{
Zelai Jin, Fuqiang Zhang \\ School of Mechanical and Automotive Engineering, Shanghai University of Engineering Science, Shanghai, China \\ Email: 546006571@qq.com
}

How to cite this paper: Jin, Z.L. and Zhang, F.Q. (2018) Analysis of Spatial Sensitivity Based on Electrostatic Monitoring Technique in Oil-Lubricated System. Open Access Library Journal, 5: e4969.

https://doi.org/10.4236/oalib.1104969

Received: October 8, 2018

Accepted: October 27, 2018

Published: October 30, 2018

Copyright $\odot 2018$ by authors and Open Access Library Inc.

This work is licensed under the Creative Commons Attribution International License (CC BY 4.0).

http://creativecommons.org/licenses/by/4.0/

\section{(c) (1) Open Access}

\begin{abstract}
In this paper, for the detection of wear particles in lubricating oil roads, by analyzing the particle belt motor, a mathematical model of the oil circuit electrostatic sensor considering the dielectric constant is proposed. At the same time, the concept of spatial sensitivity and visual field is introduced. The influencing factors of the position of the charged abrasive particles, the axial length of the sensor and the radial radius were studied respectively. The influence of each variable on the sensitivity was obtained, and the accuracy of the mathematical model was also verified. The simulation results show that reducing the radial radius of the sensor can effectively increase the spatial sensitivity of the electrostatic sensor; the larger the axial length $L$ of the electrode, the higher the sensitivity, and the more uniform the cross-sectional sensitive field distribution; in the axial and radial directions, the larger the shaft diameter ratio, the more sensitive the sensor is and the more sensitive it is.
\end{abstract}

\section{Subject Areas \\ Mechanical Engineering}

\section{Keywords}

Wear Particles, Mathematical Model, Spatial Sensitivity, Field of View, Shaft Diameter Ratio

\section{Introduction}

In the past, bearings in industrial rotating machinery caused arcing due to arc voltage and shaft current, and in the actual operation, it was impossible to ob- 
serve the mechanism of electric corrosion in real time, and the motor was easily damaged. Non-contact monitoring of the contact damages sites and products of the mechanism using electrostatic induction technology [1] [2]. The principle is based on the metal contact pair which is generated on the surface during contact, scratching, falling off, crack propagation, and formation of a white layer and a phenomenon of trace static. This technology enables real-time monitoring of the surface condition of the part, which gives the maintenance plan ample preparation time and makes predictive maintenance possible.

Aero engine Lubricating Oil Road Abrasive Monitoring is the monitoring of bearings, gears and other lubricating components of engines and wind turbine gears, and predicts the impending failure of lubricating components, thereby effectively predicting the life of components in order to protect the engine and gearbox, safety, saving maintenance costs and maximizing economic benefits. Therefore, it is one of the important components of the engine and its gearbox condition monitoring system.

Oil analysis is an effective means of monitoring wear and tear. When the engine is operating with the gearbox, it is often found in the lubricating oil that the lubricating parts of the engine and the gearbox begin to wear. For example, the lubricating parts of the engine and the gearbox are slid, fatigued or creeped, and the small bucks will peel off the substrate to form abrasive particles and flow with the lubricating oil. Therefore, the formation of abrasive particles is the result of failure of the friction surface, and the abnormal amount or large abrasive particles found in the oil monitoring indicate excessive wear or fatigue failure of the components in the engine and the gearbox.

In the past, the oil monitoring of the machine was to collect oil samples on site, and went to the laboratory to complete the analysis. After the analysis, the data was sent to the on-site maintenance personnel and decision makers. Due to the large amount of time required to transfer oil samples and data, it takes several hours to complete an oil sample analysis, and up to two months. According to the survey, $50 \%$ of the off-line analytical oil samples showed no problems, and $45 \%$ of the offline analytical oil samples showed that the failure was about to occur, and only $5 \%$ detected serious problems. In this way, not only does it consume a lot of manpower and material resources, but it is also difficult to guarantee the timeliness of the data. According to the National Aeronautics and Space Administration (NASA) study, the oil-abrasive monitoring method is more reliable than the vibration analysis method widely used in aeronautical engines in the wear failure analysis method. The wear state can be directly obtained by monitoring and analyzing the abrasive grain information [1] [2]. Electrostatic sensors are a new type of sensor for abrasive particle monitoring in aircraft engine oil systems. Compared with the traditional abrasive particle monitoring technology, real-time online monitoring can be realized, and the sensor structure is simple to install and convenient. The oil system electrostatic sensor was first developed by the British company Stewart Hughes and has been applied to 
the F-35 engine lubricating oil road surface monitoring system [3].

The research on the basic characteristics of electrostatic sensors is mainly focused on the gas-solid two-phase flow detection method. Gajewski, Yan et al. [4] [5] [6] used simulated analysis and experimental methods to measure the concentration and velocity of transported particles, while $\mathrm{Xu}$ et al. proposed an improved electrostatic sensor measurement model. The model does not exhibit specific mathematical expressions, using finite element software for simulation analysis [7] [8]. Huang et al. established a point charge model on the lube road and verified that the electrical signal is similar to the theoretical analysis voltage output of the analog monitoring experimental station. Mao et al. improved the mathematical model of the electrostatic sensor by equivalently equating the point charge into a ring shape, and verified the correctness of the mathematical model in the calibration experiment.

From the current research status at home and abroad, Lubricating Oil Road Particle Static Monitoring Technology [9] [10] [11] can detect component degradation earlier than traditional vibration and temperature monitoring methods, and can provide real-time monitoring information for PHM. Although experimental studies have shown that the waveform of the monitoring voltage is related to the nature of the charge carried by the particles, the electrostatic sensor can distinguish the abrasive grains of the buckling block and the non-metallic material, and qualitatively obtain the level of the monitoring voltage and the particle size. The conclusion about the size, however, lacks a theoretical basis to explain the relationship between the induced voltage waveform and the charged characteristics of the abrasive particles, and lacks research on the relationship between the abrasive charging characteristics and the abrasive material. Therefore, it is necessary to improve the electrostatic monitoring of the lubricating oil particles. The physical model, and using Coulomb's law and Gauss's theorem to establish a mathematical model of the particle electrostatic monitoring system, so that it can clearly indicate the relationship between the amount of electricity carried by the particles and the output of the electrostatic monitoring system and the factors affecting the relationship; Monitor the quantitative relationship between sensor spatial sensitivity and particle size, sensor radius, and axial length.

\section{Electrostatic Sensor}

\subsection{Electrostatic Sensor Structure and Induction Mechanism}

In order to verify the input-output relationship between charged particles and sensors, to understand the sensor mechanism and study the sensor characteristics, it is necessary to establish the physical and mathematical models of the sensor. The physical structure model of the electrostatic sensor is shown in Figure 1. The sensor consists of a ring probe, an insulating layer and a metal shield. The lubricating oil flows through a pipe made of an insulating material, and the annular probe installed at the inner wall of the insulating pipe is used to sense an electrostatic signal in the fluid, and the metal shielding layer is used to shield 


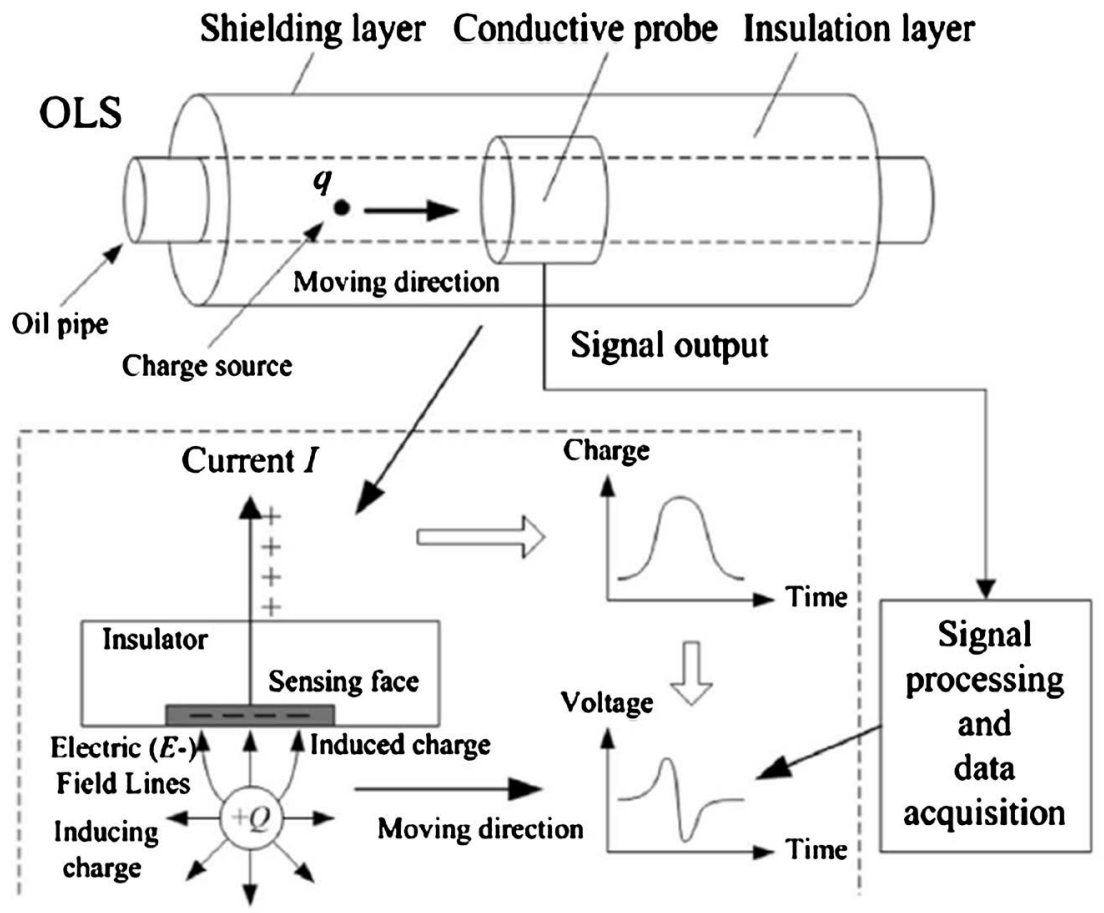

Figure 1. Schematic diagram of the electrostatic sensor and sensing mechanism.

external interference.

The mobile charge inside the electrostatic sensor probe can move freely in any direction. When charged particles pass through the electrostatic sensor, the moving charge within the sensor moves under the influence of external charges until they reach the surface of the sensor probe. The movement of charge in the sensor causes current flow through the signal line and the signal can be measured by the signal conditioner. The magnitude of the induced charge generated can be obtained by the acquisition circuit. The amount of induced charge on the electrode is amplified by the charge amplifier, converted into an analog voltage signal, and then converted into a digital signal by an AID conversion circuit and stored in a computer for analysis and processing.

The electrostatic sensor has a ring shape and a rod shape. The advantage of the ring probe is that it does not interfere with the flow field and is simple to install. The disadvantage is that it is not sensitive to charged particles passing through the center of the fluid, and is not suitable for the case where the diameter of the fluid is large. The rod-shaped probe is usually mounted on the side of the fluid conduit and inserted into the fluid. The advantage is that it can sense the charged particles in the center of the fluid. The disadvantage is that it is not easy to install, it needs to be perforated in the fluid pipeline, and the probe may have a certain influence on the internal fluid.

\subsection{Electrostatic Sensor Mathematical Model}

When the lubricating oil flows in the pipe, it generates static electricity, which affects the safety of the mechanical equipment. Scholars have proposed a num- 
ber of computational models for oil flow charging, with which the degree of oil flow can be predicted. However, there are many factors affecting the electrochemical reaction involved in the interface of the tubing wall, and the mechanism of action between the influencing factors is very complicated. The proposed model often contains some variables that are difficult to determine in the actual situation, which affects the model. Promote the application. Based on the mathematical model of the electrostatic sensor based on the principle of point charge electrostatic induction, Professor Yan emphasizes the dielectric constant in the lubricating oil. Under the condition of the known velocity distribution of the oil in the pipeline, the sensitivity characteristics of the electrostatic sensor are studied. In Figure 1, the electrostatic effect of the charged charge on the charged particles is not counted. Due to Coulomb's law, the electric field strength at any point on the point charge electrode is:

$$
E=\frac{q}{4 \pi \varepsilon d^{2}}
$$

where $q$ is the size of the induced charge and $d$ is the distance that the point charge corresponds to the electrostatic field generated on the probe. The dielectric constant of the lubricating medium is:

$$
\varepsilon=\varepsilon_{0} \varepsilon_{r}
$$

where $\varepsilon_{0}$ is the permittivity of free space and $\varepsilon_{r}$ is the relative permittivity. The electric flux density $D$ at a certain point in free space is the number of flux lines on a curved surface perpendicular to the flux line divided by the area of the surface, calculated as follows:

$$
D=\varepsilon E
$$

The induced charge $Q$ of the entire probe is equal to the flux passing through the closed surface where the probe is located.

$$
Q=\oint_{s} D \cdot \mathrm{d} S
$$

where $\mathrm{d} S$ is a microelement surface size anywhere on the sensor surface and $x$ is the radial position of charged particles. The axial position $z$ of charged particles can be obtained by:

$$
z=v t
$$

where $v$ is the speed of movement of charged particles, and $t$ is the movement time of charged particles.

\subsection{Electrostatic Sensor Spatial Sensitivity}

The sensitivity of the sensor is defined as the ratio of the output of the sensor to the input. For easy identification, the peak value of the signal pulse can be defined as the output value of the sensor. Then, the sensitivity of the electrostatic sensor can be expressed as the ratio between the induced charge and the amount of the abrasive particles when the abrasive grains pass through the center section of the electrode. Formula can be expressed as: 


$$
S_{p}=\left|\frac{Q}{q}\right|
$$

Through the analysis of mathematical models, combined with the above derivation, the closed surface formed by $-L / 2$ to $L / 2$ utilizes Gauss's law, and the sensitivity generated by the entire probe is given by:

$$
\begin{gathered}
S_{p}=\left|\frac{Q}{q}\right|=\frac{R}{2 \pi} \int_{0}^{\pi} \frac{R-x \cos \phi}{F^{2}(x, \phi)}\left\{\frac{z+L}{\left[(z+L)^{2}+F^{2}(x, \phi)\right]^{\frac{1}{2}}}-\frac{z-L}{\left[(z-L)^{2}+F^{2}(x, \phi)\right]^{\frac{1}{2}}}\right\} \mathrm{d} \phi \\
F(x, \phi)=\left(R^{2}+x^{2}-2 R x \cos \phi\right)^{0.5}
\end{gathered}
$$

where $R$ is the radius of the probe and $L$ is half the axial length of the probe. $Q$ is the original charge of the point charge and $q$ is the size of the induced charge.

\subsection{Electrostatic Sensor Input and Output Characteristics}

For electrostatic sensors, the input is the induced charge in the sensing region, which can be measured using a suitable device, and the corresponding output is the voltage signal that is collected and processed by the electrostatic sensing system.

Figure 2 shows the actual equivalent circuit model of the electrostatic sensor electrode. According to Kirchhoff's law of charge

$$
\frac{\mathrm{d} q(t)}{\mathrm{d} t}=C \frac{\mathrm{d} u_{i}(t)}{\mathrm{d} t}+\frac{u_{i}(t)}{R}
$$

where $R=R_{e} \cdot R_{i} /\left(R_{e}+R_{i}\right), C=C_{e}+C_{i}+C_{c} . C_{e}, R_{e}$ are the equivalent capacitance and insulation resistance of the electrode, respectively, the equivalent input capacitance and input impedance of the interface circuit, which is the distributed capacitance of the cable, which is the induced power on the electrode. If the initial condition is zero, a Laplace transform on the above equation can be obtained:

$$
\frac{U_{i}(s)}{Q(s)}=\frac{s R}{1+s R C}
$$

where $U_{i}(s)$ is the Laplace transform of the output voltage $u_{i}(t)$ of the interface, $Q(s)$ is the Laplace of the electrostatic sensor output induced charge
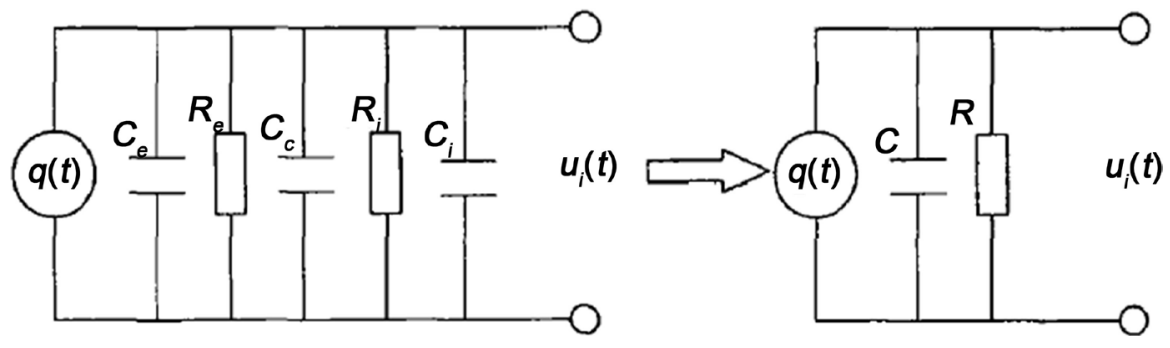

Figure 2. Electrostatic sensor equivalent circuit. 
$q(t)$. If the condition $|j w R C| \ll 1$ is satisfied, then Equation (10) can be simplified to:

$$
U_{i}(j w)=j w R Q(j w)
$$

Then the time domain response is:

$$
u_{i}(t)=R \frac{\mathrm{d} q(t)}{\mathrm{d} t}
$$

Equation (12) shows that the input voltage of the interface circuit is proportional to the rate of change of induced charge on the probe (inductive current), so the interface circuit is resistive. In relatively clean environments, charge measurements from different laboratories using different instruments may result in different absolute values due to cables, charge amplifiers, connectors, and the like. However, the output voltage is always proportional to the coefficient $R_{r^{*}}$

\subsection{Field of View}

Since the electrostatic sensor detects charge only in the sensing area of the detector, the "field of view" is introduced as a basic and important parameter of the electrostatic sensor. The field of view is defined as the measurable maximum spatial range detected by the electrostatic sensor as it induces charge through the sensing area of the sensor probe. The electrostatic sensor cannot detect an out-of-range charge portion and is therefore a "blind zone" for the sensor. The field of view is an important parameter that characterizes the range of the sensor and can also be used to optimize the design of the electrostatic sensor.

It is assumed that the inductive point charge passes through the electrostatic sensor at a constant radial position at a constant velocity $v_{c}$. When the level first rises above the baseline, the peak starts from $p_{1}$, and when the level returns to the baseline level, the peak ends at $p_{2}$. The duration from $p_{1}$ to $p_{2}$ is considered to be the charge duration through the probe $t_{p}$. Therefore, the static television field $m_{p}$ at the corresponding radial position can be calculated by the following equation [11].

$$
m_{p}=v_{c} t_{p}
$$

Duration $t_{p}$ can be used $t_{p}=t_{p 2}-t_{p 1}$ to calculate. The total field of view in the axial direction of the sensor is a collection of this field. View all corresponding radial positions. Since the field of view of the electrostatic sensor in the radial direction is limited to the inside of the sensor, the field of view of the electrostatic sensor is generally referred to as the axial range. Due to the symmetrical nature of the ring probe, the field of view is the same on both sides of the center of the probe. Therefore, the survey is only simplified for one side of the sensor. The entire field of view can then be obtained and can be doubled by a half range.

\section{Electrostatic Sensor Spatial Sensitivity Analysis}

\subsection{Axial Length Affects Sensitivity}

The axial length of the electrode has an effect on the electric field distribution of 
the sensitive space of the sensor. Therefore, in order to determine the length of the electrode, it is necessary to investigate the influence of the length of the electrode on the distribution of the sensitivity of the electrostatic sensor. The sensitivity of the sensor with the axial length of the electrode of $4 \mathrm{~mm}, 10 \mathrm{~mm}, 20 \mathrm{~mm}$ and $40 \mathrm{~mm}$ was simulated by MAXWELL. The calculation results are shown in Figure 3.

Figure 3 shows the variation of the sensitivity of the center cross section of each probe in the radial direction in the case where the axial length of the probe is different. It can be seen from the figure that when the axial length of the electrode is increased, the value of the sensitivity of the radial position on the cross section is increasing, but not in a proportional relationship. As the axial length of the electrode increases, the sensitivity change parameter $S$ gradually decreases, that is, the sensitivity tends to be uniform, and the sensitivity of the sensor is also increased, that is, the longer the electrode, the better. However, excessively increasing the length of the electrode can cause significant spatial filtering effects, and excessively increasing the axial length of the electrode can make the sensor less robust and the sensor probe susceptible to bending. Under the condition of ensuring the sensitivity and sensitivity of the sensor, the characteristics of the integrated sensor are finally selected to be $10 \mathrm{~mm}$ in axial length.

\subsection{Effect of Ring Probe Radius on Sensitivity}

The installation position of the inlet ring sensor determines that the ring should be as close as possible to the pipe wall so that it does not affect the air flow field. In order to investigate whether the sensor has an influence on the sensitivity in the inlets of different diameters, the electrodes with diameters of $20 \mathrm{~mm}, 50 \mathrm{~mm}$, $80 \mathrm{~mm}$ and $100 \mathrm{~mm}$ were simulated. Before the simulation, the finite element model needs to be modified to adjust the air domain between the electrode casing and the electrode. The calculation results are shown in Figure 4.

\subsection{Sensitivity Analysis in the Radial Direction}

In order to further discover the influence of the radius and axial length of the

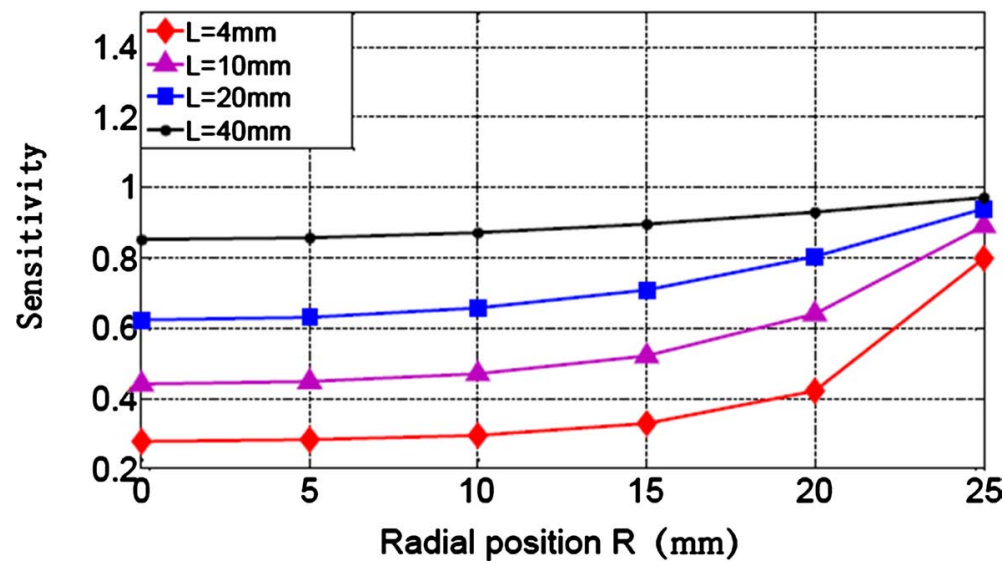

Figure 3. Radial center section sensitivity of electrodes with different axial lengths. 
electrostatic sensor on the sensitivity, select $z=0, x=0.01$, and use matlab to obtain the influence of the two on the sensitivity.

As shown in Figure 5, in the radial direction, the range of the radius of the electrostatic sensor $0.01<R<0.05$ is selected, and the range of the axial length $0<L<0.1$ is the sensitivity of the probe at different positions in the space. Figure 5 shows the sensitivity distribution of sensors with different radii and different axial lengths. From the figure, the size of the induced probe radius has little effect on the sensitivity distribution trend of the induced probe, and the sensitivity space increases slightly with the increase of the radius. According to the same distance from the side of the probe, the sensitivity of the probes with different radii is distributed along the axial position. It can be seen from the figure that in the same distance from the side, at the same axial position, the radius

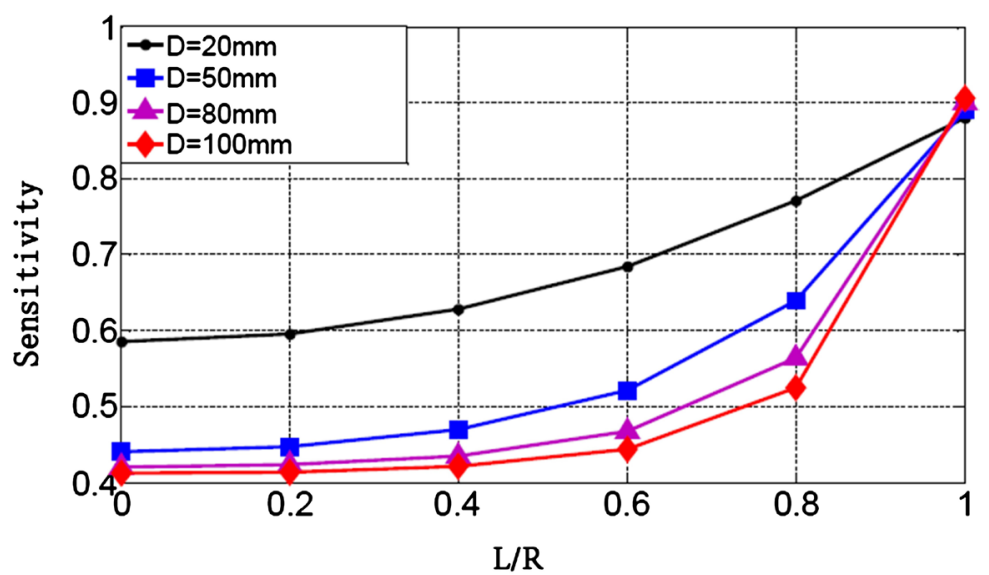

Figure 4. Radial center section sensitivity of different diameter electrodes.

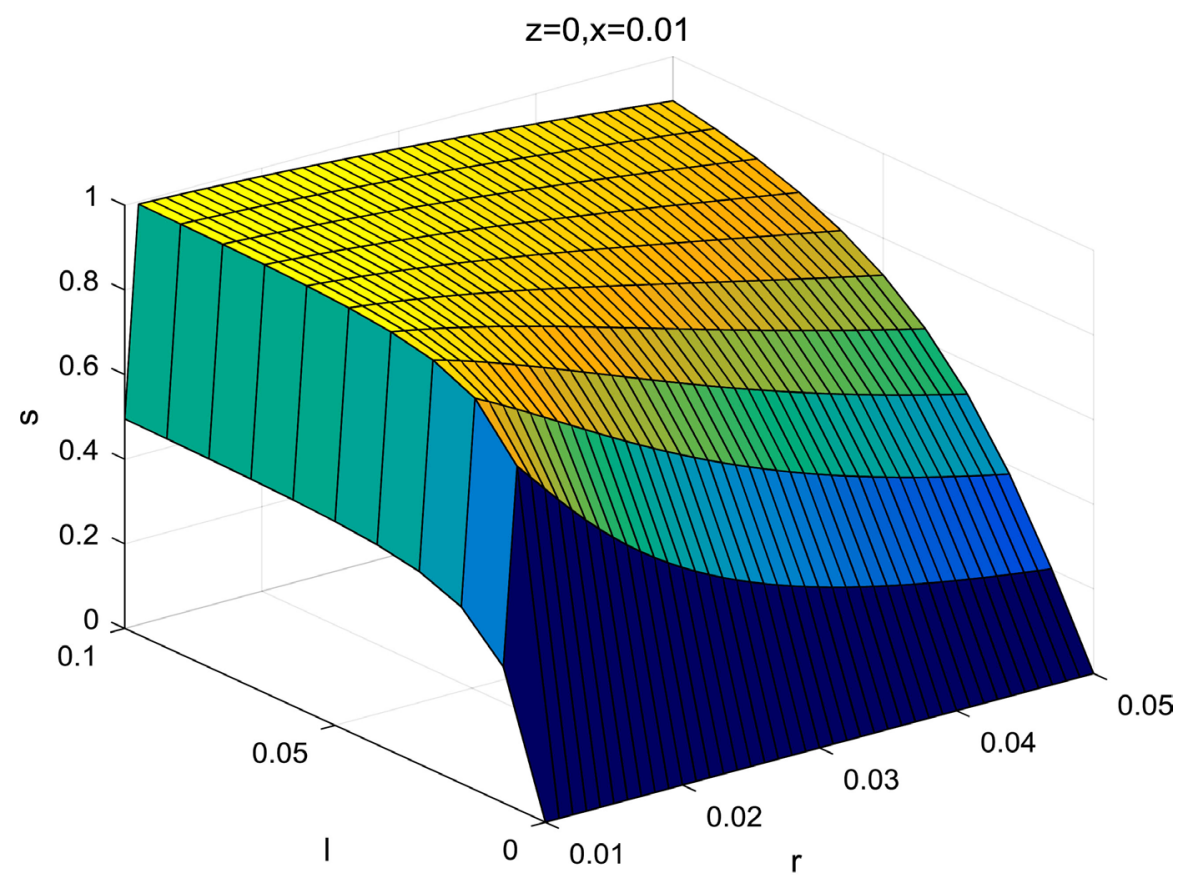

Figure 5. Effect of $L$ and $R$ on sensitivity in radial $x=0.01$. 
is large. The sensitivity of the sensor is relatively large.

The radial sensitivity distribution when two groups were selected ( $L=0.05, R$ $=0.05 ; L=0.0125, R=0.05)$ is clearly as shown in Figure 6 . The sensitivity gets the maximum value at $x=0$, which is the center of the pipe. The closer to the tube wall sensitivity, the maximum is obtained. The larger the shaft diameter ratio, the greater the sensitivity.

\subsection{Sensitivity Analysis in the Axial Direction}

As shown in Figure 7, in the axial direction, the range of the radius of the electrostatic sensor is selected, and the range of the axial length is the sensitivity of the probe at different positions in the space. Figure 7 shows the sensitivity

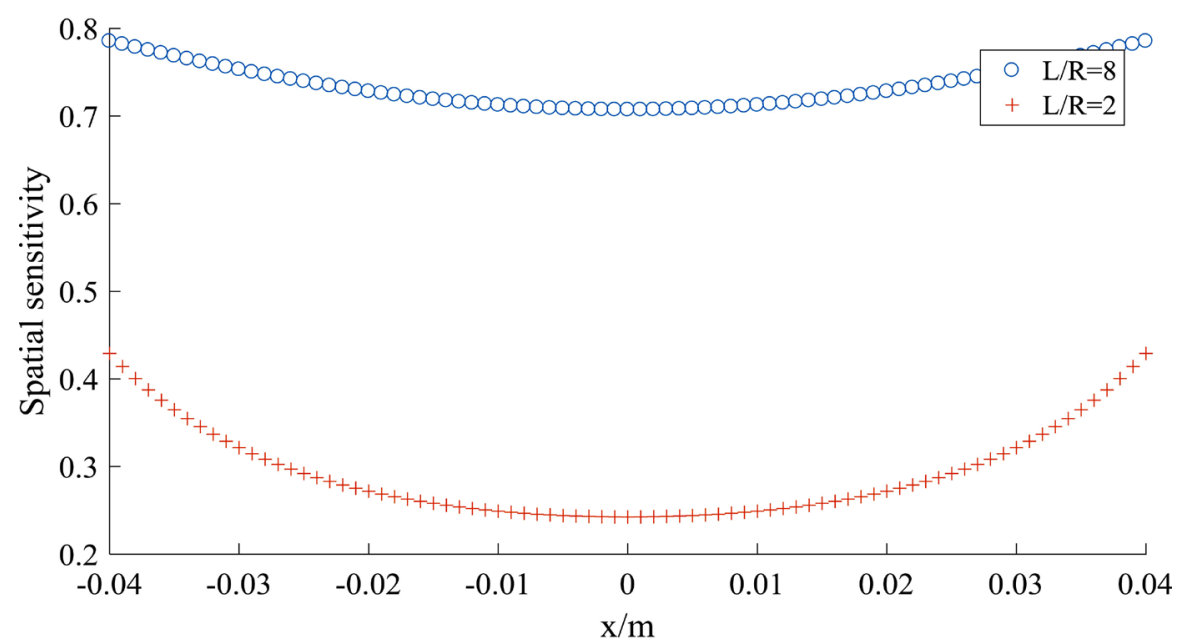

Figure 6. Sensitivity radial distribution at $z=0$.

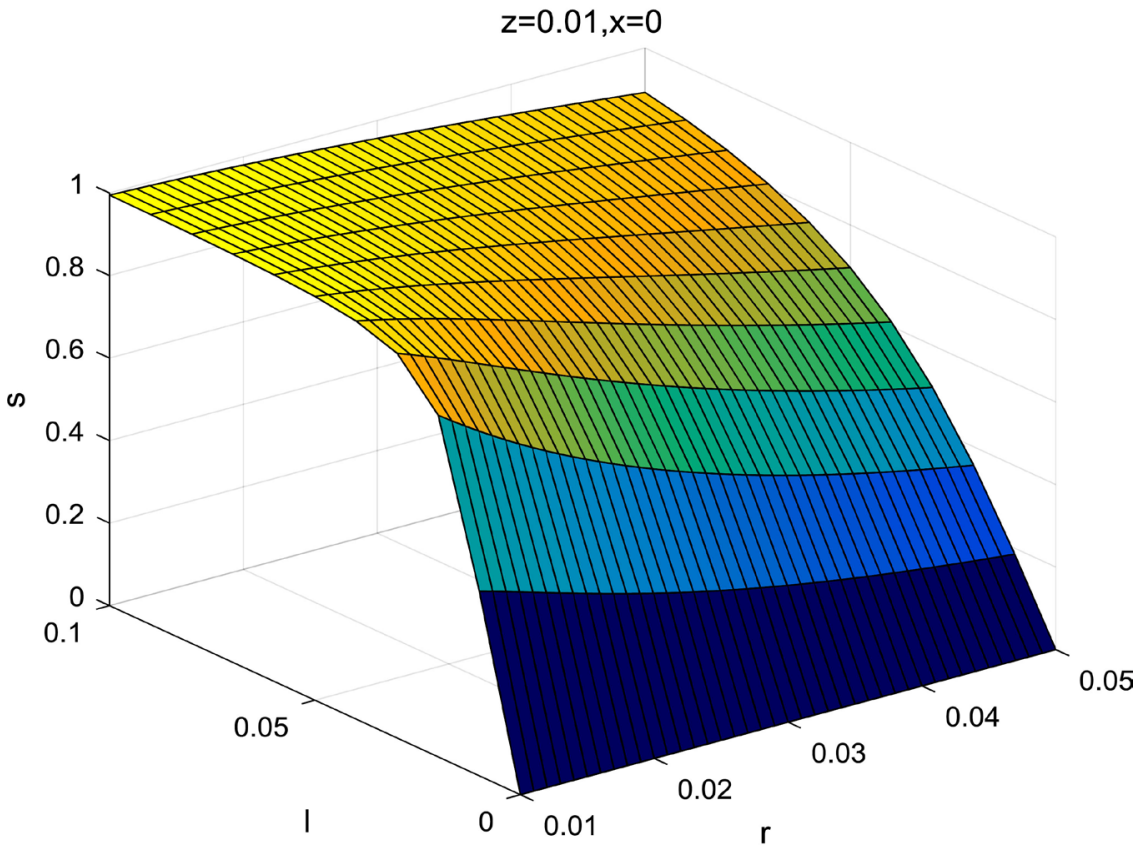

Figure 7. The influence of $L$ and $R$ on the sensitivity in the axial direction at $z=0.01$. 
distribution of sensors with different radii and different axial lengths. It is apparent in the picture that the longer the axial length, the greater the sensitivity.

The axial sensitivity distribution when two groups are selected $(L=0.02, R=$ $0.01 ; L=0.02, R=0.04$ ) is clearly as shown in Figure 8 .

As can be seen from Figure 8, the electrostatic sensor is located at the same radial length, and the distance from the sensor to the $z$-axis is further, that is, the closer to the sensor wall, the higher the spatial sensitivity of the electrostatic sensor, and vice versa, the lower the spatial sensitivity. At the same time, as the radial length of the electrostatic sensor becomes longer, the spatial sensitivity of the sensor is lower at the same radial position. It can also be understood that the greater the ratio of the axial length to the radial diameter of the electrostatic sensor, the higher the spatial sensitivity.

Figure 5, Figure 6, and Figure 7 confirm each other, thus demonstrating the correctness of the spatial sensitivity formula, and thus the mathematical model established in this paper is correct. In addition, the spatial sensitivity of the electrostatic sensor is also affected by factors such as the moving speed of the charged abrasive particles, the thickness of the insulated pipe and its dielectric constant, and these influencing factors are independent of the physical properties of the electrostatic sensor itself.

\section{Spatial Sensitivity Distribution Characteristics}

According to the mathematical model of the electrostatic sensor, when $x=0$, the electrostatic sensor obtains the maximum sensitivity at $x=0$, which is of great significance for the study of axial sensitivity. The factors that affect the spatial sensitivity of the electrostatic sensor in the axial direction are mainly the position of the charged abrasive particles and the axial length and radial radius of the sensor. The axial length and radius influence of the sensor have been obtained by simulation. Since the electrostatic sensor has a radial symmetry characteristic, when the charged abrasive grain passes through the electrostatic sensor sensing

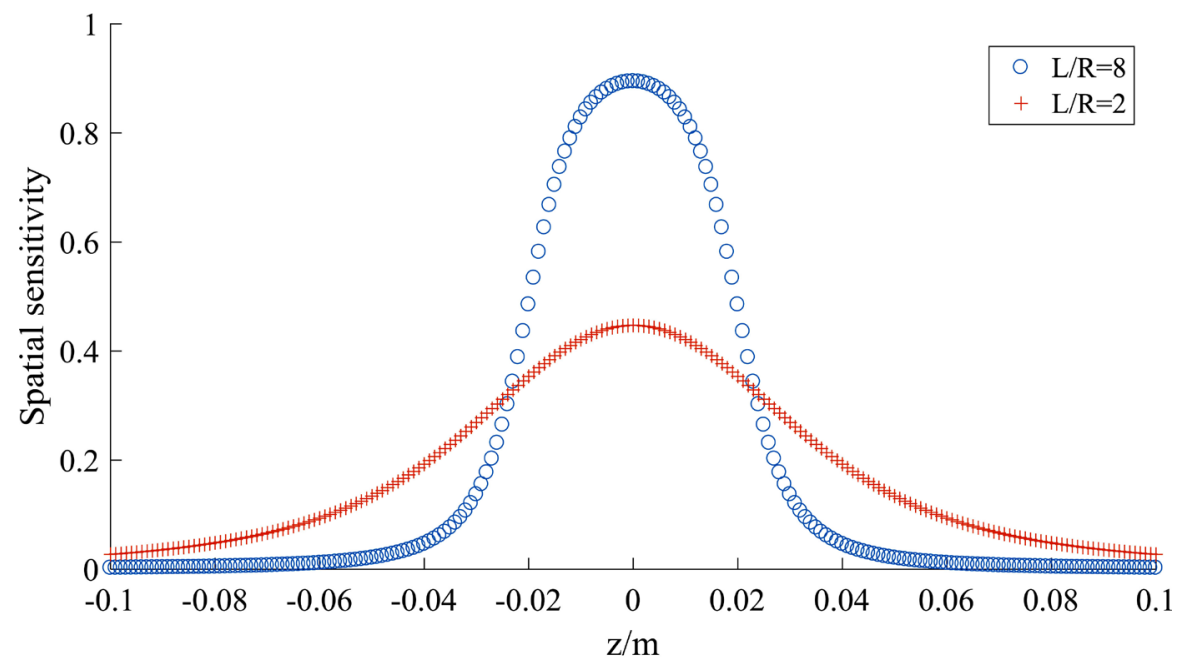

Figure 8. Axis distribution of sensitivity at $\mathrm{x}=0$. 
area at a certain position in the radial direction of the sensor, the axial length of the sensor Under the condition of L constant, the sensitivity of the sensor is only related to the relative position of the charged abrasive particles in the radial direction. For the convenience of observation, let $x=0, L=0.01 \mathrm{~m}$, and obtain the axial distribution of the electrostatic sensitivity of the electrostatic sensor as shown in Figure 9.

Inside the probe, it can be seen from Figure 9 that the closer the radial position is to the probe wall, the higher the spatial sensitivity and the sensitivity has a maximum at the central axis of the pipe, consistent with the previous conclusions. If the trajectory of the point charge (abrasive grain) in the radial position cannot be known, only the sensing area of the sensor near the axis can be selected, that is, the sensor probe of the sensor is embedded in the insulating layer, and only the static sensitivity of the oil is allowed. The flow is changed in a gently changing area, and the output of the sensor can be approximated regardless of the influence of the radial position. This has important guiding significance for the design of the sensor.

\section{Conclusions}

1) Reducing the radial radius of the sensor can effectively increase the spatial sensitivity of the electrostatic sensor. The closer the charged particles are to the wall surface of the pipe in the radial position, the higher the spatial sensitivity. The installation of the electrostatic sensor close to the monitoring surface during installation can effectively improve the electrostatic sensor sensitivity. The sensitive field of the sensor is greater than the axial length of the probe, so no sources of interference can occur in the sensitive field.

2) The larger the axial length $L$ of the electrode, the higher the sensitivity, the

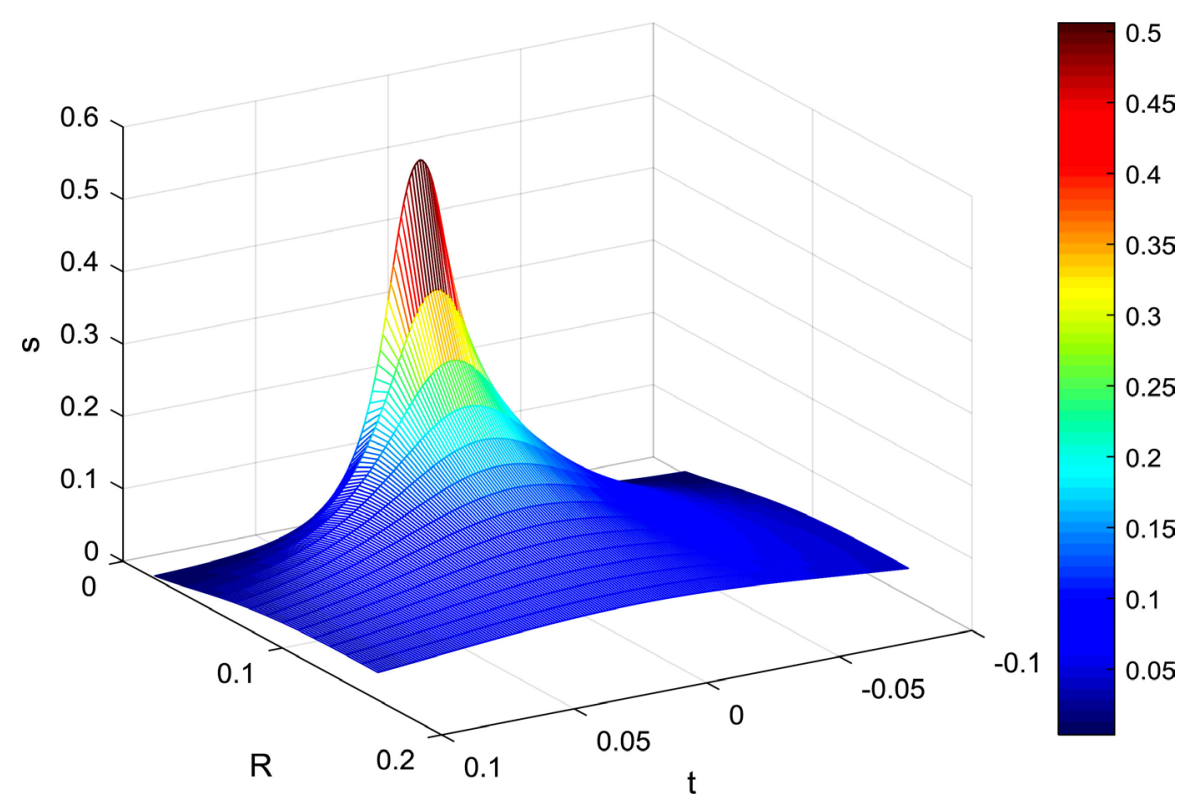

Figure 9. Axial sensitivity distribution of electrostatic sensors. 
more uniform the distribution of the sensitive field of the section, and the corresponding range of the axial sensitive space; but the electrode is too long, the sensor is facing the fluid space due to the electrostatic filter effect of the electrostatic sensor. The high frequency signal of the structure loses its responsiveness, causing significant spatial filtering effects, making the sensor less robust and the probe susceptible to bending. The axial length of the electrostatic sensor must be chosen reasonably.

3) The larger the axial diameter ratio in the axial direction and the radial direction, the more sensitive the sensor is and the more sensitive it is. The value of the shaft diameter ratio should be as large as possible.

\section{Conflicts of Interest}

The authors declare no conflicts of interest regarding the publication of this paper.

\section{References}

[1] Harvey, T.J., Wood, R.J.K. and Powrie, H.E.G. (2007) Electrostatic Wear Monitoring of Rolling Element Bearings. Wear, 263, 1492-1501. https://doi.org/10.1016/j.wear.2006.12.073

[2] Wen, Z., Ma, X. and Zuo, H. (2014) Characteristics Analysis and Experiment Verification of Electrostatic Sensor for Aero-Engine Exhaust Gas Monitoring. Measurement, 47, 633-644. https://doi.org/10.1016/j.measurement.2013.09.041

[3] Powrie, H.E.G. and Fisher, C.E. (1999) Engine Health Monitoring: Towards Total Prognostics. 1999 IEEE Aerospace Conference, Snowmass, 7 March 1999, 11-20. https://doi.org/10.1109/AERO.1999.789759

[4] Gajewski, J.B. (1996) Monitoring Electrostatic Flow Noise for Mass Flow and Mean Velocity Measurement in Pneumatic Transport. Journal of Electrostatics, 37, 261-276. https://doi.org/10.1016/0304-3886(96)00016-2

[5] Gajewski, J.B. (1999) Electrostatic Flow Probe and Measuring System Calibration for Solids Mass Flow Rate Measurement. Journal of Electrostatics, 45, 255-264. https://doi.org/10.1016/S0304-3886(98)00048-5

[6] Yan, Y., Byrne, B., Woodhead, S. and Coulthard, J. (1995) Velocity Measurement of Pneumatically Conveyed Solids Using Electrodynamic Sensors. Measurement Science and Technology, 6, 515-537. https://doi.org/10.1088/0957-0233/6/5/013

[7] Xu, C., Zhou, B. and Wang, S. (2010) Dense-Phase Pneumatically Conveyed Coal Particle Velocity Measurement Using Electrostatic Probes. Journal of Electrostatics, 68, 64-72. https://doi.org/10.1016/j.elstat.2009.10.003

[8] Wang, S., Xu, C., Li, J., et al. (2016) An Instrumentation System for Multi-Parameter Measurements of Gas-Solid Two-Phase Flow Based on Capacitance-Electrostatic Sensor. Measurement, 94, 812-827. https://doi.org/10.1016/j.measurement.2016.09.010

[9] Liu, R.C., Zuo, H.F., Sun, J.Z., et al. (2017) Simulation of Electrostatic Oil Line Sensing and Validation Using Experimental Results. Tribology International, 105, 15-26. https://doi.org/10.1016/j.triboint.2016.09.026

[10] Mao, H., Zuo, H., Huang, W., et al. (2016) Mathematical Modelling and Calibration Experiment of New Electrostatic Sensor in Aviation. Acta Aeronautica et Astronau- 
tica Sinica, 37, 2242-2250.

[11] Chen, Z.X., Zuo, H.F., Zhan, Z.J., et al. (2012) Study of Oil System Oil-Line Debris Electrostatic Monitoring Technology. Acta Aeronautica et Astronautica Sinica, 33, 446-452. 